\title{
RF-based motion estimation using non-rigid image registration techniques: in-silico and in-vivo feasibility
}

\author{
Brecht Heyde*, Martino Alessandrini*, Ling Tong*§, Jan D’hooge*‡ \\ ${ }^{*}$ Cardiovascular Imaging and Dynamics, University of Leuven, Leuven, Belgium \\ $\S$ Center for Biomedical Imaging Research, Tsinghua University, Beijing, China

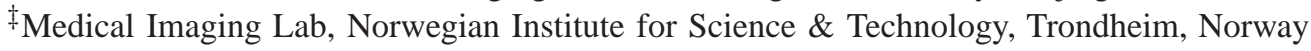 \\ Email: brecht.heyde@med.kuleuven.be
}

\begin{abstract}
US deformation techniques can roughly be divided in block matching (BM) and non-rigid image registration (NRIR). Motion can be extracted from the radio-frequency (RF) signals, from their envelope, or from the B-mode data. RF-based BM is known to outperform B-mode tracking in a small displacement setting, whereas NRIR has only been applied to B-mode data. The aim of this study was to test the feasibility of RF-based NRIR in-silico and in-vivo. First, synthetic 2D images of a phantom with a soft inclusion undergoing an axial compression $(0.25 \%)$ were simulated. Its performance was assessed by varying the inclusion thickness (range: $2-20 \mathrm{~mm}$ in $2 \mathrm{~mm}$ steps) and stiffness (resulting strain range: $0.50 \%-1.50 \%$ in $0.25 \%$ steps). Both RF and envelope tracking were better at identifying smaller and more subtle inclusions compared to B-mode tracking (down to $8 \mathrm{~mm}$ and $6 \mathrm{~mm}$ resp.). Furthermore, when tracking the RF instead of their envelope, inclusion borders were more sharply defined (border size $2.57 \mathrm{~mm}$ vs $4.88 \mathrm{~mm}, p<0.001$ ) and strain errors in the inclusion were lower $(0.08 \%$ vs $0.10 \% ; p<0.05)$. Next, NRIR was used to track the septum of a healthy volunteer from high frame rate US recordings $(436 \mathrm{~Hz})$, and compared against a recent $\mathrm{RF}$-based $\mathrm{BM}$ method. In-vivo tracking revealed that RF-based BM and RF-based NRIR performed similarly, both producing physiological axial velocity and strain curves. The lateral components could only be estimated reliably using NRIR.
\end{abstract}

Index Terms-Strain, Non-rigid registration, RF, B-mode

\section{INTRODUCTION}

Ultrasonic (US) myocardial deformation imaging has received much attention as an imaging technique to quantify regional cardiac function non-invasively [1]. Several technical advances now allow 2D images to be acquired at a very high frame rate (order of $500 \mathrm{~Hz}$ ), which may grant insight into new areas of myocardial mechanics and blood flow [2]. Compared to conventional imaging this also poses challenges for motion/deformation estimation techniques given that the inter-frame motion is significantly smaller.

US deformation techniques can roughly be divided in block matching (BM) and non-rigid image registration (NRIR). From a data processing perspective, motion can be extracted from the radio-frequency (RF) signals, from their envelope, or from the B-mode data (after subsampling and scanconversion).

For small displacements, RF-based BM is known to outperform B-mode tracking due to the high frequency content of the RF signals [3]. However, BM typically requires an a-posteriori regularization step and a least-squares fitting procedure to obtain reliable strain maps [4]. NRIR on the other hand incorporates prior information during the motion estimation process and allows computing the strain analytically. However, NRIR has thus far been applied on B-mode data only, with the exception of a recent publication in intravascular US [5].

The aim of this study was therefore to test in an in-silico setting whether RF-based NRIR was feasible and if it would outperform B-mode based NRIR. Its performance was also evaluated in-vivo by tracking the septum of a healthy volunteer from high frame rate US recordings.

\section{MATERIALS AND METHODS}

\section{A. Motion and strain estimation strategy}

In this work, tracking is performed using either the RF signals, their envelope or the B-mode data:

B-mode: A previously developed NRIR technique based on a B-spline free-form deformation model was used to compute motion between subsequent B-mode images [6]. Speckle patterns were tracked by minimizing an energy term based on the sum-of-squared differences and a bending energy penalty term. The weight of the latter term was set empirically by visually optimizing the tracking result. A total of four B-spline grid refinement steps were used, halving the span every step.

Envelope: Tracking was performed in a similar fashion by tracking the envelope $e(x)$ of the RF signals instead. Envelope detection was performed for every RF line $l(x)$ as:

$$
e(x)=\sqrt{l(x)^{2}+l_{H}(x)^{2}}
$$

with $l_{H}(x)$ the Hilbert transform of the associated RF line. More grid refinement steps (six) were used to account for the relatively larger axial motions (in absolute samples) compared to B-mode tracking. Please note that compared to the B-mode images, envelope images are highly anisotropic, i.e. consisting of few but densily sampled RF lines. The B-spline span was therefore only refined in the axial direction.

RF: In order to estimate motion using the RF signals, images were first pre-aligned based on the detected envelope using the registration schedule described above. Next, an additional registration step was performed using the RF signals. In other words, a mixed image pyramid was constructed based on two image types (the derived envelope images at the top six levels, and the original RF images at the bottom 


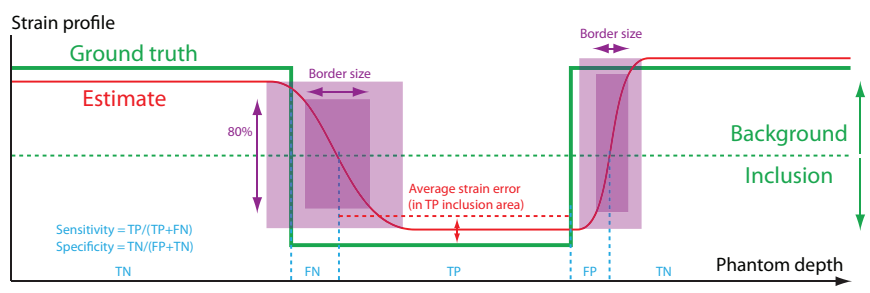

Fig. 1. Schematic overview of the different quality measures when identifying in-silico inclusions: sensitivity, specificity, border size and average strain error

and final level). The pre-alignment stage was used to avoid peak hopping which would occur if the RF images would be registered directly.

Strain estimation: After registration, strain $\varepsilon_{\mathbf{N}}$ in any given (unit) direction $\mathbf{N}$ was computed analytically using the deformation gradient tensor $\mathbf{F}$ of the B-spline transform as [7]:

$$
\varepsilon_{\mathbf{N}}=\sqrt{\mathbf{N}^{\mathrm{T}} \cdot \mathbf{F}^{\mathrm{T}} \cdot \mathbf{F} \cdot \mathbf{N}}-1
$$

\section{B. In-silico tracking performance}

Synthetic 2D RF images of a phantom $(60 \times 60 \mathrm{~mm})$ containing a soft middle layer were simulated $\left(3.4 \mathrm{MHz}, 75^{\circ}\right.$ sector). The phantom was fixed at the bottom and underwent an inter-frame axial compression of $0.25 \%$. In order to assess the spatial resolution of NRIR, the thickness of the soft layer was reduced from $20 \mathrm{~mm}$ to $2 \mathrm{~mm}$ (in $2 \mathrm{~mm}$ steps), and its stiffness was decreased to obtain a range of axial strain values $(0.5 \%$ to $1.5 \%$, in $0.25 \%$ steps). For each of these stiffnessthickness combinations, a pair of RF images (150 lines $\times 9377$ axial samples) was simulated from which the envelope and Bmode images were generated [8]. This process was repeated 10 times to account for the stochastic nature of the RF data. As such, a total of 500 simulated RF image pairs were obtained (5 stiffness options $\times 10$ thickness options $\times 10$ realisations).

The NRIR technique was used to compute motion from either the B-mode, envelope or RF image pairs as outlined in Sect.II-A. Axial strain values were subsequently estimated by evaluating Eq. (2) in the ROI and every pixel was classified as background or inclusion using the average reference strain difference between the soft layer and its surroundings as a cutoff. The softer inclusion was considered found when the pixel-based sensitivity and specificity were both larger than 0.8. The border zone around the inclusion layer was defined as the thickness in which $80 \%$ of the strain difference between both neighboring layers could be recovered. Finally, the absolute average axial strain error in the identified inclusion was also calculated. These metrics were computed first for every realisation (Fig. 1), and then averaged over all realisations to obtain performance indices for each stiffnessthickness combination.

\section{In-vivo tracking performance}

The performance of the RF-based NRIR technique was evaluated in-vivo by tracking the septum of a healthy volunteer. RF images $(176$ lines $\times 8994$ axial samples $\times 920$ frames) were acquired using the ULA-OP system [9] able to perform parallel receive and transmit beamforming $(2.0 \mathrm{MHz}$

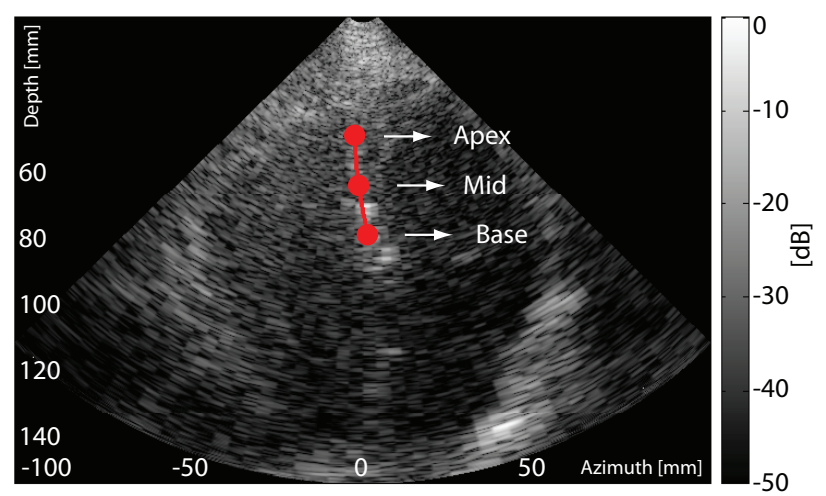

Fig. 2. Example B-mode image of a healthy volunteer. Strain was estimated along the curved red line over the septum.

transducer, $90^{\circ}$ sector scan, 4 multi-line transmissions, 16 multi-line acquisitions, $436 \mathrm{~Hz}$ frame rate). Axial and lateral strain was computed from the analytical displacement field according to Eq. (2). They were estimated along a curved line following the septum as shown in Fig. 2.

Additionally, its performance was contrasted against that of a fast state-of-the-art cross-correlation based RF block matching technique (1D kernel size $5 \lambda=3.8 \mathrm{~mm}, 70 \%$ axial window overlap, 2D search region size $4.7 \mathrm{~mm} \times 5$ lines; [10]). After tracking, a-posteriori regularisation was performed by median filtering the obtained velocities both in space ( 9 axial $\times 5$ lateral samples) and in time (7 frames). Axial and lateral strain was then obtained using the classical tissue Doppler imaging pipeline [11]. First, strain rate was calculated as the slope of the regression line of all velocities within a certain axial window (in this case 9 samples; [4]). Next, strain was estimated by temporally integrating the strain rate curves.

For both techniques, only the first cardiac cycle was considered to generate the strain curves. Unfortunately, no simultaneous ECG recordings were available. End-diastole was therefore identified using the axial velocity profiles [12]. Strain curves were drift corrected by equally distributing the strain offset over the cardiac cycle.

\section{RESULTS}

An overview of the detected inclusions is shown in Fig. 3. Some example strain profiles through the phantom are highlighted in Fig. 4. When a threshold of 0.8 was used for both the sensitivity and specificity, respectively 17, 36 and 33 inclusion combinations could be detected when tracking was based on B-mode, envelope or RF signals. The corresponding border size was respectively $7.19 \mathrm{~mm}, 4.88 \mathrm{~mm}$ and $2.57 \mathrm{~mm}$. The average absolute axial strain error in the inclusion area was respectively $0.22 \%, 0.10 \%$ and $0.08 \%$.

Fig. 5 summarizes the in-vivo septal tracking results as velocity profiles along a curved M-mode. Fig. 6 provides the corresponding velocity curves and derived strain curves at different locations along the septum.

\section{DISCUSSION AND CONCLUSION}

In this paper, two different experiments were used to assess the performance of RF-based NRIR. 

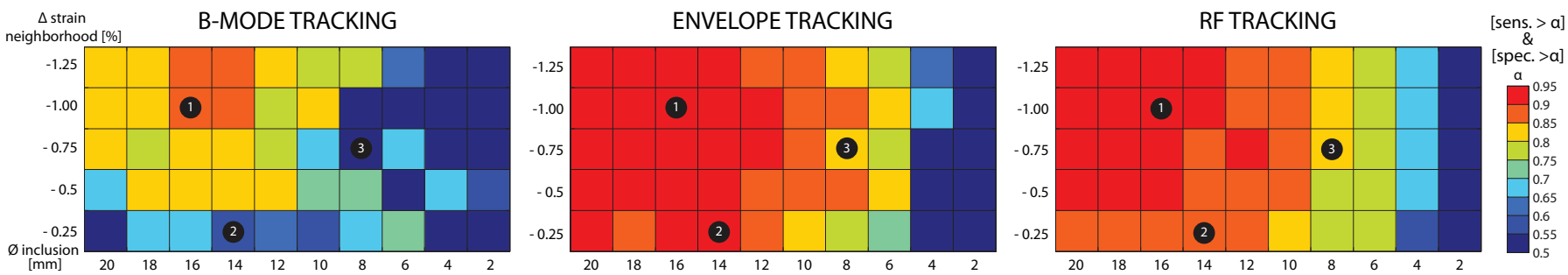

Fig. 3. In-silico tracking performance expressed in terms of a combined sensitivy and specificity threshold to detect the softer inclusion in the evaluated multilayer phantom combinations. Numbers refer to the example strain profiles highlighted in Fig. 4.
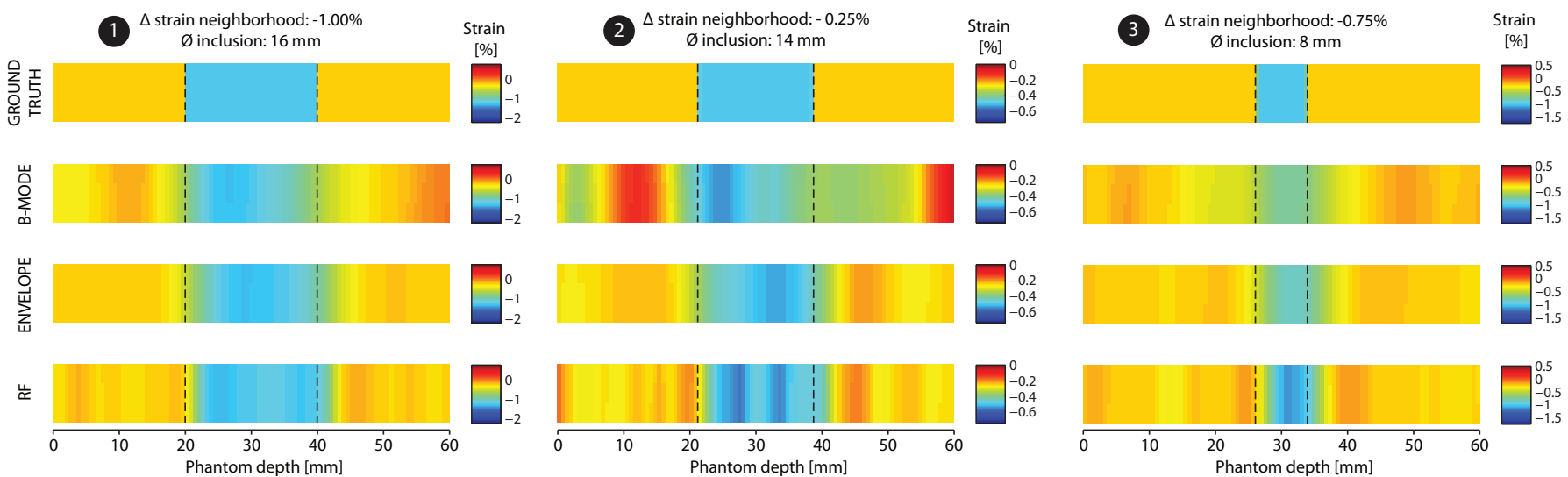

Fig. 4. Example strain profiles through the in-silico phantom obtained by NRIR through tracking the B-mode data, the envelope or the RF signals. The numbers refer to inclusion stiffness-thickness combinations in Fig. 3. The dotted lines indicate the exact position of the layer interfaces.

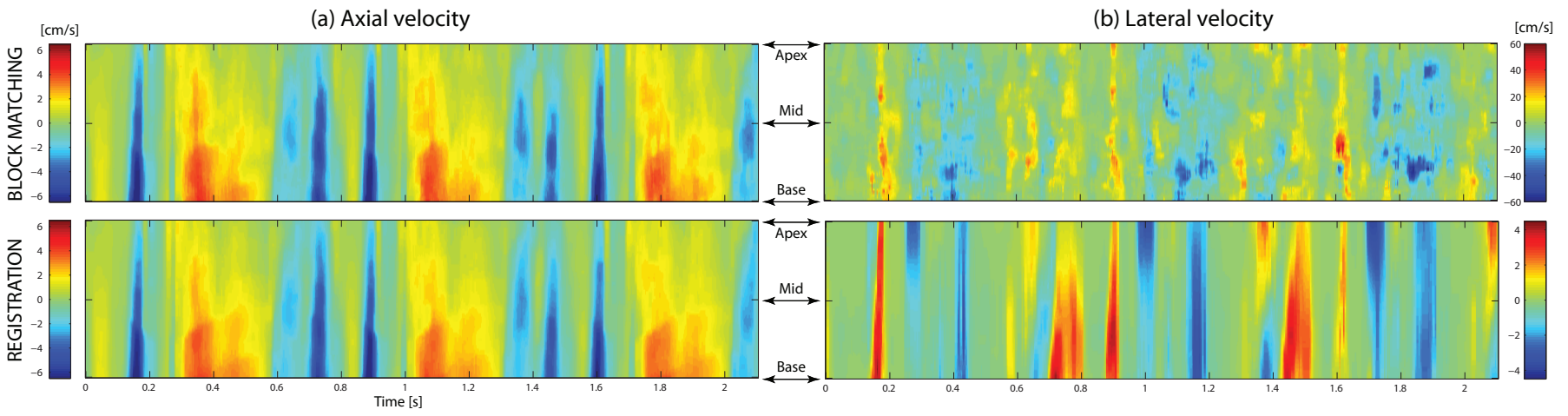

Fig. 5. (a) Axial and (b) lateral velocity curved M-mode profiles through the septum (see Fig. 2) of a healthy volunteer obtained by BM or NRIR.

In the in-silico setting it was found that tracking in beamspace prior to subsampling was superior to B-mode tracking with approximately two thirds of the evaluated stiffnessthickness combinations being recovered as opposed to only one third in case of B-mode tracking. Using the sensitivity and specificity criteria employed in Fig. 3, envelope tracking performed slightly better than RF tracking, both in terms of absolute numbers (respectively 36 versus 33 distinguishable inclusions) and in terms of difficulty (the smallest observable layer thickness was down to $6 \mathrm{~mm}$ versus $8 \mathrm{~mm}$ respectively).

However, the other quality measures favored tracking with an extra RF refinement step: the average absolute axial strain error in the inclusion was smaller $(0.08 \%$ versus $0.10 \%$, $\mathrm{p}<0.05$ ), and the inclusion was more sharply defined since the border size was smaller $(2.57 \mathrm{~mm}$ versus $4.88 \mathrm{~mm}$, $\mathrm{p}<0.001)$. The latter effect is also clearly visible in Fig. 4 when comparing rows 3 and 4 . It should be noted that although the interface was better visible with RF tracking (particularly in case of smaller inclusions, e.g. Fig. 4 column 3), there also seemed to be a consistent localisation bias for all techniques, with the position of the right inclusion border being overestimated towards the right. This may be due to the initial B-spline grid which was symmetrically positioned over the image in combination with the unidirectional but asymmetrical compression (the phantom was fixed on the right). Given that RF tracking resulted in a more pronounced (but slightly shifted) soft inclusion, its performance was therefore intrinsically underrated in comparison with envelope tracking when the inclusion sensitivity and specificity were used as quality criteria. 

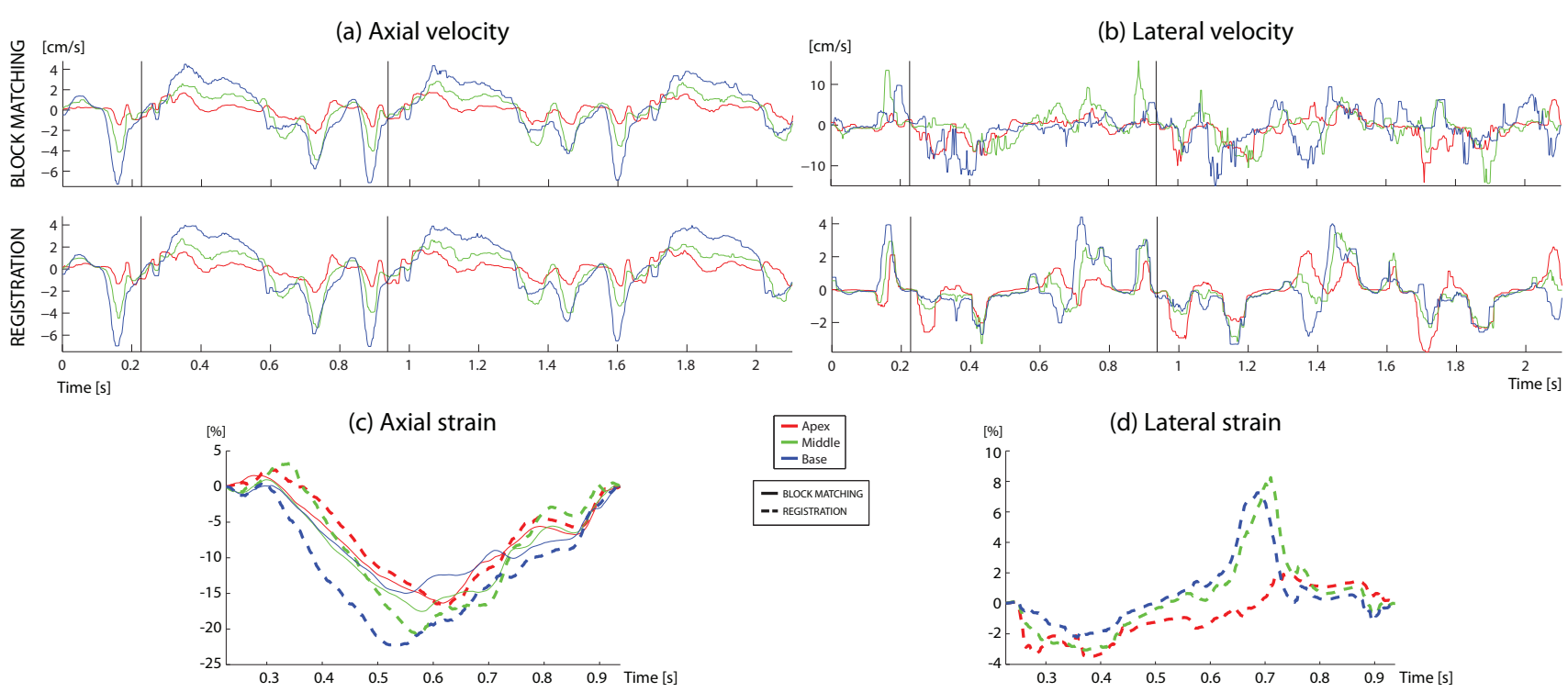

Fig. 6. (a) Axial and (b) lateral velocity and corresponding (c) axial strain and (d) lateral strain curves at three locations of the septum (see Fig. 2) from an US recording of a healthy volunteer obtained by BM and NRIR. Strain curves are extracted from the cardiac cycle highlighted by black vertical lines.

When tracking the in-vivo dataset, both BM and NRIR produced very similar curved M-mode axial velocity profiles along the septum in terms of amplitude, spatial distribution and temporal trend (Fig. 5a). The obtained curves had a physiological shape with clear S-, E- and A-waves (Fig. 6a). Qualitatively, the axial NRIR velocities appeared slightly smoother. The derived axial strain curves of both techniques were similar in shape (Fig. 6c) and had normal amplitudes.

From Figs. $5 \mathrm{~b}$ and $6 \mathrm{~b}$ it is evident that the lateral velocity plots for BM were too high in amplitude. They were also more noisy compared to the axial component. This is not surprising as it is intrinsically more difficult to extract the lateral component due to the lower frequency (and US resolution) in this direction. No BM-derived strain curves were therefore extracted in Fig. 6d as they would not be meaningful. The curves obtained with NRIR on the other hand were less noisy and were cyclic in nature. This is most likely due to the fact that NRIR imposes regularisation during the motion optimisation process and can therefore better cope with more difficult tracking conditions in the lateral direction. Furthermore, strain is less sensitive to noise in the motion estimates as it can be derived analytically as opposed to requiring numerical derivations in BM. Nevertheless, it should be noted that the lateral NRIR velocity appeared quantitized. Upsampling the RF lines in the lateral direction prior to registration may improve these results.

In conclusion, RF- and envelope-based NRIR were better at identifying smaller and more subtle in-silico inclusions compared to B-mode tracking. Furthermore, the inclusion borders were more sharply delineated and strain errors were lower when tracking the RF signals instead of their envelope. Invivo tracking revealed that RF-based BM and RF-based NRIR performed similarly in the axial direction, both producing physiological axial velocity and strain curves. The lateral components could only be estimated reliably using NRIR.

\section{ACKNOWLEDGMENTS}

This work was supported by the Research Fund KU Leuven.

\section{REFERENCES}

[1] R. Jasaityte, B. Heyde, and J. D'hooge, "Current state of threedimensional myocardial strain estimation using echocardiography," $J$ Am Soc Echocardiogr, vol. 26, no. 1, pp. 15-28, 2013.

[2] M. Cikes, L. Tong, G. Sutherland, and J. D'hooge, "Ultrafast cardiac ultrasound imaging," J Am Coll Cardiol: Cardiovasc Imaging, vol. 7, no. 8, pp. 812-823, 2014.

[3] W. Yu, P. Yan, A. Sinusas, K. Thiele, and J. Duncan, "Towards pointwise motion tracking in echocardiographic image sequences - comparing the reliability of different features for speckle tracking," Med Image Analysis, vol. 10, no. 4, pp. 495-508, 2006.

[4] D. Fleming, X. Xia, W. McDicken, G. Sutherland, and L. Fenn, "Myocardial velocity gradients detected by doppler imaging," $\mathrm{Br} J$ Radiol, vol. 67, no. 799, pp. 679-688, 1994.

[5] M. Richards and M. Doyley, "Non-rigid image registration based strain estimator for intravascular ultrasound elastography," Ultrasound Med Biol, vol. 39, no. 3, pp. 515-533, 2013.

[6] B. Heyde, R. Jasaityte, D. Barbosa, V. Robesyn, S. Bouchez, P. Wouters, F. Maes, P. Claus, and J. D'hooge, "Elastic image registration vs speckle tracking for 2D myocardial motion estimation: a direct comparison invivo," IEEE Trans Med Imaging, vol. 32, no. 2, pp. 449-459, 2013.

[7] L. Malvern, Introduction to the mechanics of a continuous medium. Englewood Cliffs, NJ: Prentice-Hall, 1969.

[8] H. Gao, H. Choi, P. Claus, S. Boonen, S. Jaecques, G. Van Lenthe, G. Van Der Perre, W. Lauriks, and J. D'hooge, "A fast convolutionbased methodology to simulate 2-D/3-D cardiac us images," IEEE Trans Ultrason Ferroelectr Freq Control, vol. 56, no. 2, pp. 404-409, 2009.

[9] P. Tortoli, L. Bassi, E. Boni, A. Dallai, F. Guidi, and S. Ricci, "ULA-OP: an advanced open patform for ultrasound research," IEEE Trans Ultrason Ferroelectr Freq Control, vol. 56, no. 10, pp. 2207-2216, 2009.

[10] J. Luo and E. Konofagou, "A fast normalized cross-correlation calculation method for motion estimation," IEEE Trans Ultrason Ferroelectr Freq Control, vol. 57, no. 6, pp. 1347-1357, 2010.

[11] J. D'hooge, A. Heimdal, F. Jamal, T. Kukulski, B. Bijnens, F. Rademakers, L. Hatle, P. Suetens, and G. Sutherland, "Regional strain and strain rate measurements by cardiac ultrasound: principles, implementation and limitations," Eur J Echocardiogr, vol. 1, no. 3, pp. 154-170, 2000.

[12] G. Sutherland, T. Kukulski, J. Voigt, and J. D'hooge, "Tissue Doppler echocardiography: future developments," Echocardio, vol. 16, no. 5, pp. 509-520, 1999. 